\title{
X-ray ferromagnetic resonance spectroscopy
}

\author{
G. Boero a) and S. Rusponi \\ Ecole Polytechnique Fédérale de Lausanne (EPFL), CH-1015 Lausanne, Switzerland \\ P. Bencok \\ European Synchrotron Radiation Facility (ESRF), F-38043 Grenoble, France
}

R. S. Popovic, H. Brune, and P. Gambardella

Ecole Polytechnique Fédérale de Lausanne (EPFL), CH-1015 Lausanne, Switzerland

(Received 2 June 2005; accepted 6 September 2005; published online 5 October 2005)

\begin{abstract}
We present a method to measure continuous-wave ferromagnetic resonance (FMR) spectra based on the core-level absorption of circularly polarized $\mathrm{x}$ rays. The technique is demonstrated by using a monochromatic x-ray beam incident on an yttrium-iron-garnet sample excited by a microwave field at $2.47 \mathrm{GHz}$. FMR spectra are obtained by monitoring the x-ray absorption intensity at the photon energy corresponding to the maximum of the magnetic circular dichroism effect at the iron $L_{2,3}$ edges as a function of applied magnetic field. The x-ray FMR signal is shown to be energy dependent, which makes the technique element sensitive and opens up new possibilities to perform element-resolved FMR in magnetic alloys and multilayers. (C) 2005 American Institute of Physics.
\end{abstract}

[DOI: $10.1063 / 1.2089180]$

Techniques based on ferromagnetic resonance (FMR) are widely employed for the analysis of magnetic materials and thin films. ${ }^{1,2}$ Recent developments have seen the implementation of FMR detection schemes alternative to conventional inductive methods, such as those based on magnetic resonance force microscopy ${ }^{3,4}$ and Kerr effect. ${ }^{5,6}$ Behind these efforts is the need to understand and optimize the dynamic response of magnetic compounds and devices of increasing complexity. ${ }^{7}$ In this context, the development of methods capable of elemental analysis represents an obvious advantage, allowing one to investigate crucial issues such as the dynamic coupling and magnetization relaxation of elemental moments in alloys, ${ }^{8-10}$ molecular compounds, exchange-coupled multilayers, ${ }^{1,2,11,12}$ as well as current-induced magnetization excitations in spin-valve structures. ${ }^{13,14}$ Very promising results in this direction have been obtained in the time domain by pump-probe x-ray magnetic circular dichroism (XMCD) experiments with sub-ns resolution, taking advantage of the element-resolving power of core-level x-ray absorption. ${ }^{15-17}$ Recently, Bailey et al. ${ }^{16}$ have shown that time-resolved XMCD allows to detect the precessional frequency of elemental magnetic moments in a ferromagnetic alloy. Here, we propose and demonstrate a method to measure continuous-wave FMR spectra by means of XMCD by studying the time-averaged $\mathrm{x}$-ray absorption signal of $\mathrm{Fe}$ in $\mathrm{YIG}\left(\mathrm{Y}_{3} \mathrm{Fe}_{5} \mathrm{O}_{12}\right)$ excited by a microwave magnetic field at $\mathrm{GHz}$ frequencies.

$\mathrm{XMCD}$ is defined as the dependence of the $\mathrm{x}$-ray absorption coefficient on the relative orientation of the photon helicity $\boldsymbol{\sigma}$ with respect to the sample magnetization $\mathbf{M} .^{18}$ The $\mathrm{XMCD}$ effect scales as the scalar product $|\boldsymbol{\sigma} \cdot \mathbf{M}|$. The proposed technique exploits this dependence to detect timeaveraged variations of the $\mathrm{x}$-ray absorption signal due to the resonant excitation of precession modes that affect the projection of $\mathbf{M}$ onto its equilibrium direction, which is set parallel to $\boldsymbol{\sigma}$ by an external field $\mathbf{B}_{0}$. The experimental setup,

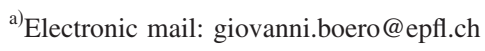

schematically shown in Fig. 1, consists of a coplanar waveguide $\lambda / 2$ resonator placed in a static magnetic field $\mathbf{B}_{0}$ that can be swept between 0 and $\pm 0.5 \mathrm{~T}$. A YIG polycrystalline slab was chosen as test sample, owing to its intense FMR response. The sample, with dimensions $1 \times 1 \times 0.05 \mathrm{~mm}^{3}$, was positioned at the center of the resonator. At its resonance frequency $\omega=2 \pi \times 2.47 \mathrm{GHz}$, the resonator produced a microwave magnetic field $\mathbf{B}_{1} \approx 0.1 \mathrm{mT}$ parallel to the sample surface with an input power of $100 \mathrm{~mW}$. The amplitude of $\mathbf{B}_{1}$ close to the resonant field, however, is not precisely known due to the feedback of the sample susceptibility on the resonator tuning. The experiment was carried out at Beamline ID08 of the European Synchrotron Radiation Facility in Grenoble by using the photon beam generated by two helical undulators with nearly $100 \%$ circular polarization rate. The intensity of the $\mathrm{x}$-ray beam was $2 \times 10^{13}$ photons/s with an energy resolution of $1.2 \mathrm{eV}$ at $700 \mathrm{eV}$, and a spot size of $0.2 \times 1 \mathrm{~mm}^{2}$ at the sample position. The beam incidence direction was aligned with $\mathbf{B}_{0}$ and set perpendicular to the sample surface. X-ray absorption spectra (XAS) were measured by recording the sample fluorescence yield by means of a Si photodiode (Eurysys-Canberra, France) as a function of the incident photon energy. X-ray FMR (XFMR) spectra were recorded at a fixed photon energy as a function of $\mathbf{B}_{0}$ in
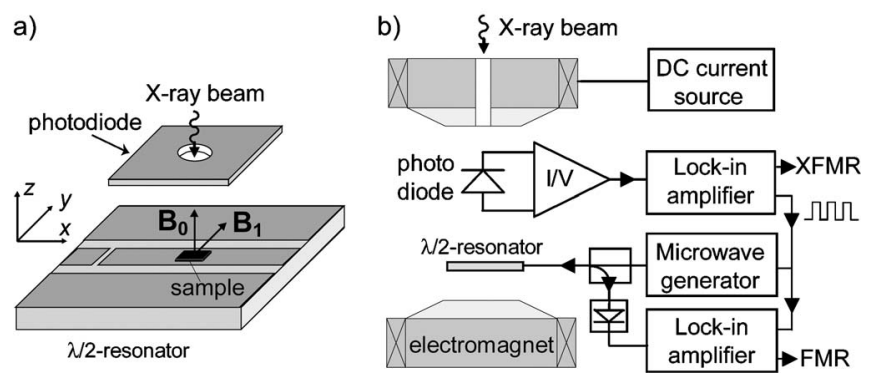

FIG. 1. Schematic representation of the XFMR setup. (a) Resonator, sample, and photodiode (not to scale). Photodiode dimensions: 22 $\times 22 \mathrm{~mm}^{2}$; through-hole diameter: $5 \mathrm{~mm}$. (b) Block diagram of the electronics. 

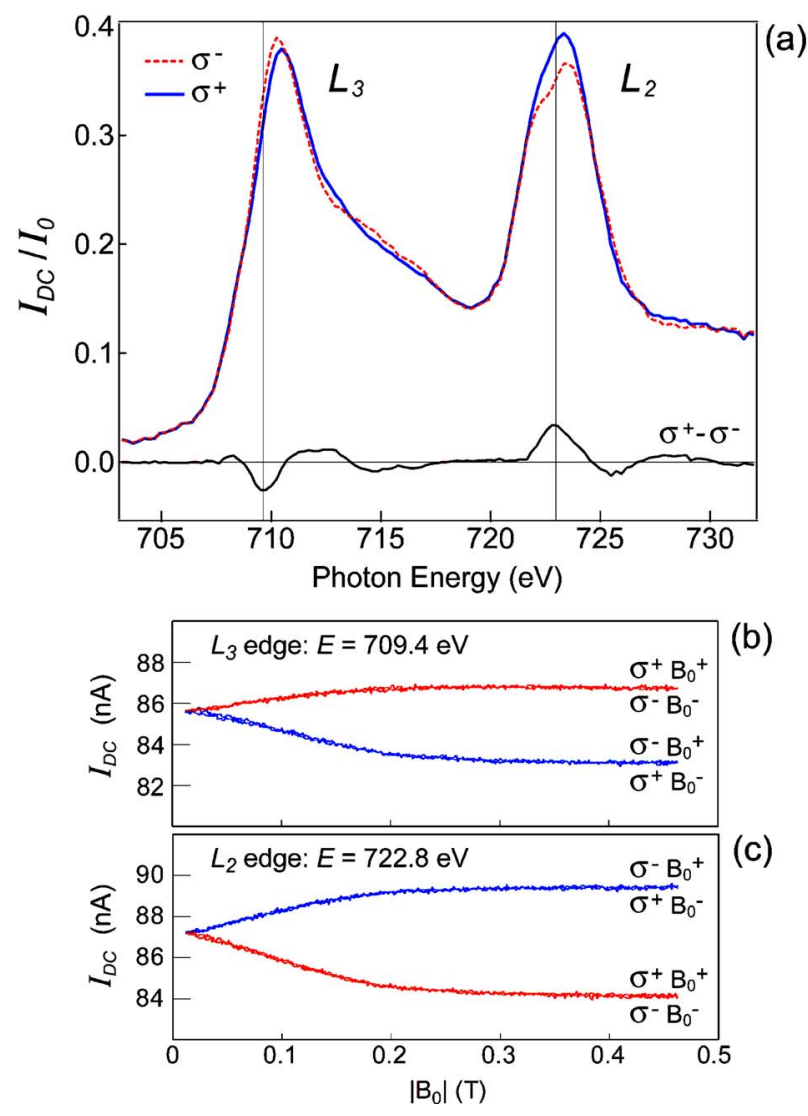

FIG. 2. (Color online) (a) XAS spectra over the $L_{2,3}$ edges of Fe recorded with photon polarization parallel $\left(\boldsymbol{\sigma}^{-}\right.$, dashed line) and antiparallel $\left(\boldsymbol{\sigma}^{+}\right.$, solid line) to $\mathbf{B}_{0}=-0.5 \mathrm{~T}$. The dc fluorescence current $I_{\mathrm{dc}}$ is shown normalized to the incident photon flux $I_{0}$, measured by the drain current of the first refocussing mirror upstream of the sample. The XMCD spectrum $\left[I_{\mathrm{dc}}\left(\boldsymbol{\sigma}^{+}\right)\right.$ $\left.-I_{\mathrm{dc}}\left(\boldsymbol{\sigma}^{-}\right) / I_{0}\right]$ is shown at the bottom. The spectra are not corrected for selfabsorption effects. (b) $I_{\mathrm{dc}} v s$. $\left|\mathbf{B}_{0}\right|$ recorded at the $L_{3}$ edge $(E=709.4 \mathrm{eV})$ and (c) $L_{2}$ edge $(E=722.8 \mathrm{eV})$.

the presence of the microwave field. The photodiode, placed at a distance of $5 \mathrm{~mm}$ in front of the sample, was operated in the photovoltaic mode and connected to a transimpedance amplifier with a gain of $10^{8} \mathrm{~V} / \mathrm{A}$ and a bandwidth of 10 $\mathrm{kHz}$. For the XFMR measurements, the microwave generator output [Fig. 1(b)] was pulse modulated at a frequency of 3.5 $\mathrm{kHz}$. The amplified photodiode signal was fed into a lock-in amplifier, which was set to measure the amplitude of the input signal at the pulse-modulation frequency. The resonator, photodiode, and the electromagnet poles were placed in a dark evacuated vessel at a pressure of $10^{-4} \mathrm{~Pa}$ and operated at room temperature.

Figure 2(a) shows the $L_{3}\left(2 p_{3 / 2} \rightarrow 3 d\right)$ and $L_{2}\left(2 p_{1 / 2}\right.$ $\rightarrow 3 d)$ core-level XAS of Fe in YIG recorded for negative $\left(\boldsymbol{\sigma}^{-}\right)$and positive $\left(\boldsymbol{\sigma}^{+}\right)$helicity of the x-ray beam with $\mathbf{B}_{0}=-0.5 \mathrm{~T}$. The configurations $\boldsymbol{\sigma}^{ \pm} \mathbf{B}_{0}^{\mp}$ and $\boldsymbol{\sigma}^{ \pm} \mathbf{B}_{0}^{ \pm}$give identical pairs of spectra, as the XMCD depends only on the relative alignment of $\boldsymbol{\sigma}$ and $\mathbf{B}_{0}$. The XMCD spectrum $\left[I_{\mathrm{dc}}\left(\boldsymbol{\sigma}^{-}\right)-I_{\mathrm{dc}}\left(\boldsymbol{\sigma}^{+}\right)\right]$indicates that the largest dichroic effects at the $L_{3}$ and $L_{2}$ edges are observed at 709.4 and $722.8 \mathrm{eV}$, respectively. Figures 2(b) and 2(c) show the absolute dc fluorescence current $I_{\mathrm{dc}}$ recorded at these energies as a function of $\mathbf{B}_{0}$ in the four $\boldsymbol{\sigma}^{ \pm} \mathbf{B}_{0}^{\mp}$ and $\boldsymbol{\sigma}^{ \pm} \mathbf{B}_{0}^{ \pm}$configurations. As expected, the XMCD reverses sign at the $L_{3}$ and $L_{2}$ edge. Note that the XMCD decreases to zero as $\mathbf{B}_{0}$ tends to zero, owing to the negligible remanent magnetization of our sample,

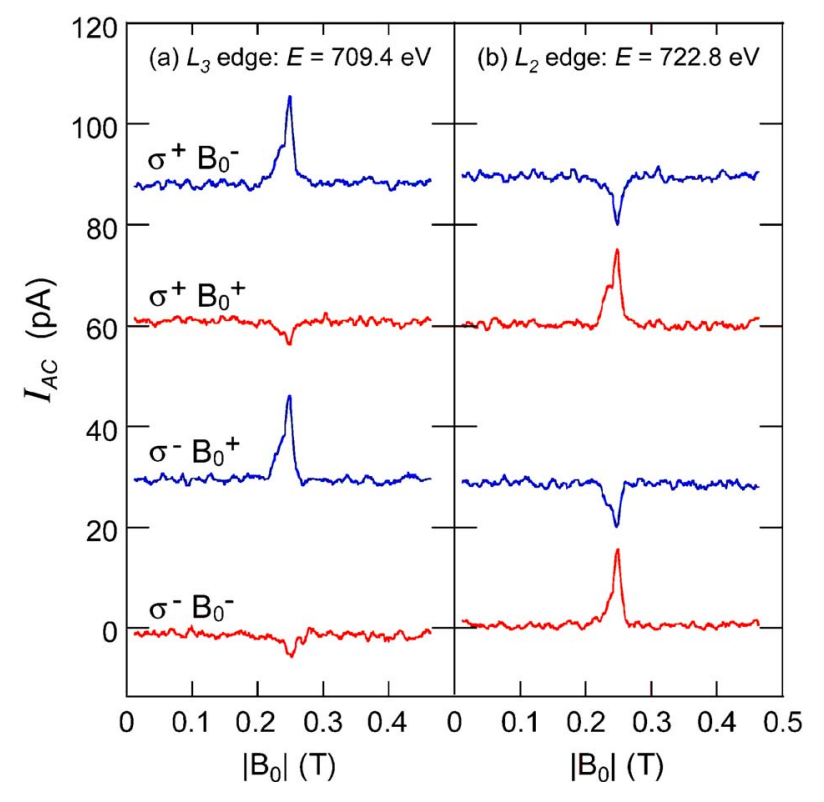

FIG. 3. (Color online) ac component of the photodiode current $\left(I_{\mathrm{ac}}\right)$ at the $\mathbf{B}_{1}$ pulse-modulation frequency as a function of $\left|\mathbf{B}_{0}\right|$ and $\boldsymbol{\sigma}$. (a) $L_{3}$ edge $(E=709.4 \mathrm{eV})$ and (b) $L_{2}$ edge $(E=722.8 \mathrm{eV})$. The spectra have been offset for clarity. Average of four sweeps, time per sweep $t_{s}=107 \mathrm{~s}$, lock-in time constant $\tau_{c}=1 \mathrm{~s}$.

while it tends to a constant value for $\mathbf{B}_{0}>0.2 \mathrm{~T}$ owing to the saturation of the magnetization.

The XFMR measurements were performed by setting the photon energy to either 709.4 or $722.8 \mathrm{eV}$ and recording the amplitude of the ac photodiode current $I_{\mathrm{ac}}$ at the pulsemodulation frequency of $\mathbf{B}_{1}$ as a function of $\mathbf{B}_{0}$. Figure 3 shows the XFMR spectra obtained for the different combinations of $\boldsymbol{\sigma}$ and $\mathbf{B}_{0}$. In all cases, we observe a peak at $\left|\mathbf{B}_{0}\right|=0.25 \mathrm{~T}$, in good agreement with the resonant field $\omega / \gamma+\mu_{0} M_{s}=0.27 \mathrm{~T}$ given by Kittel's formula for the uniform precession of the magnetization in a perpendicularly magnetized thin plate. Here, $\mu_{0} M_{s}=0.18 \mathrm{~T}$ for YIG, where $M_{s}$ is the saturation magnetization. The asymmetric character of the resonance peak and its width $\left(\Delta B_{0} \approx 10 \mathrm{mT}\right.$ at $\left.-3 \mathrm{~dB}\right)$ are attributed to imperfections of the sample surface and edges, and to variations of the demagnetizing field across the sample surface. ${ }^{19,20}$

The key feature of the spectra in Fig. 3 is the reversed sign of the XFMR peak for the $\boldsymbol{\sigma}^{ \pm} \mathbf{B}_{0}^{\mp}$ configurations with respect to $\boldsymbol{\sigma}^{ \pm} \mathbf{B}_{0}^{ \pm}$. This, together with the sign inversion at the $L_{3}$ and $L_{2}$ edge, represents compelling evidence of the $\mathrm{XMCD}$ origin and photon energy dependence of the observed signal. By comparing the amplitude of the XFMR peak $(\sim 15 \mathrm{pA})$ with the variation of $I_{\mathrm{dc}}$ in Figs. 2(b) and 2(c) corresponding to the complete reversal of $\mathbf{M}(\sim 5 \mathrm{nA})$, the variation of the $z$ component of the magnetization $M_{z}$ with respect to its static equilibrium value $M_{s}$ is estimated to be about $1 \%$. An interesting point is that the negative and positive XFMR peaks do not have the same amplitude. Part of this effect is due to the asymmetry of the $\boldsymbol{\sigma}^{ \pm}$XAS intensity at $\left|\mathbf{B}_{0}\right|=0.25 \mathrm{~T}$ with respect to the intensity level of the demagnetized state of the sample, as observed by comparing the $I_{\mathrm{dc}}$ in Fig. 2(b) and 2(c) for $\boldsymbol{\sigma}^{ \pm} \mathbf{B}_{0}^{\mp}$ and $\boldsymbol{\sigma}^{ \pm} \mathbf{B}_{0}^{ \pm}$. However, the asymmetry of the peak amplitudes in Fig. 3 is larger than the $I_{\mathrm{dc}}$ asymmetry, particularly at the $L_{3}$ edge, implying that additional effects might come into play. A possible explanation is that microwave-induced changes of the population of to AIP license or copyright, see http://apl.aip.org/apl/copyright.jsp 

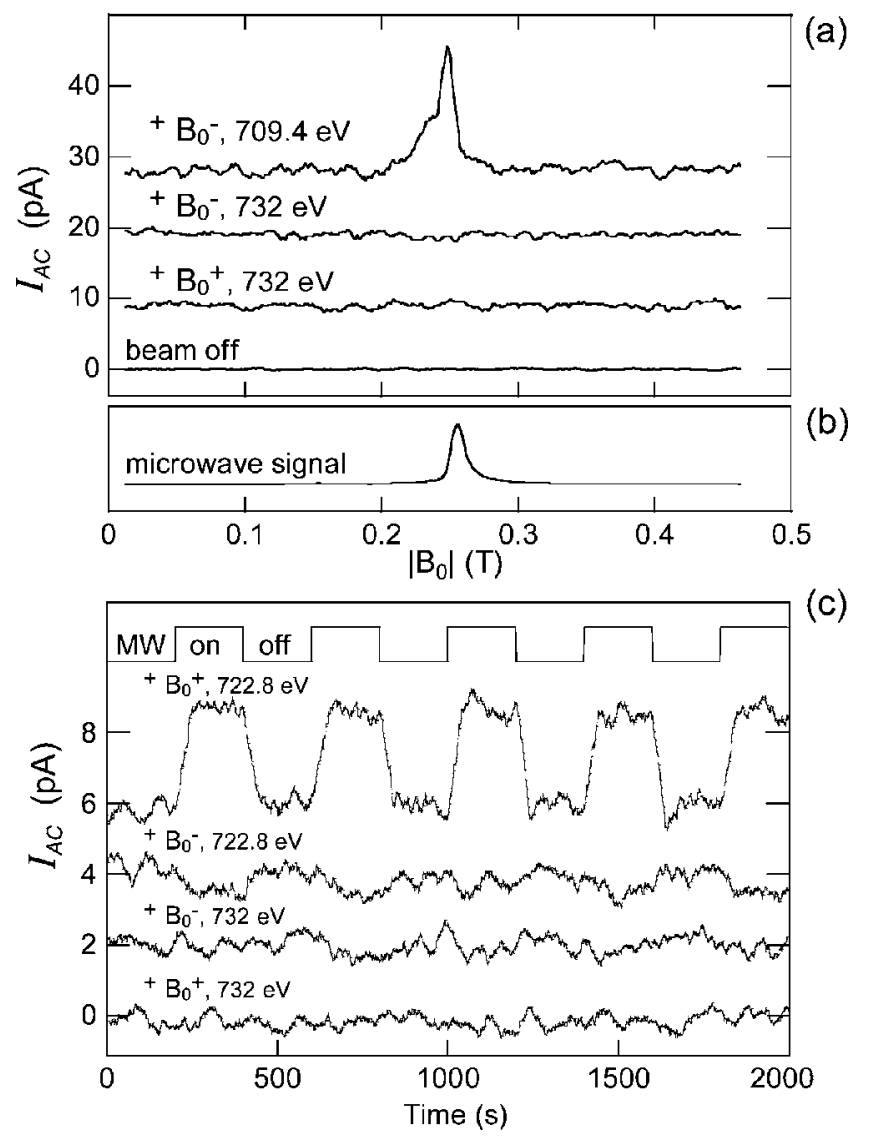

FIG. 4. (a) $I_{\mathrm{ac}}$ measured as in Fig. 3 at $709.4 \mathrm{eV}, 732 \mathrm{eV}$, and in the absence of x-ray beam. (b) Conventional FMR spectrum detected by means of the microwave bridge shown in Fig. 1. (c) $I_{\mathrm{ac}}$ vs time at $\left|\mathbf{B}_{0}\right|=0.25 \mathrm{~T}$ recorded with the microwave field switched on/off every $200 \mathrm{~s}$, lock-in time constant $\tau_{c}=20 \mathrm{~s}$. The microwave power level is about five times lower with respect to (a), resulting in a reduced signal amplitude. The spectra have been offset for clarity.

Fe $d$-states with different total angular momentum quantum number $J_{z}$ affect the XAS intensity and XFMR amplitude via the dipolar selection rules in an asymmetric fashion depending on the relative alignment of $\boldsymbol{\sigma}$ and $\mathbf{B}_{0}{ }^{21}$ Investigating the dependence of the XFMR asymmetry for larger microwave power levels than are presently available in our setup might help to elucidate the physical origin of the asymmetry.

The noise level in the XFMR spectra corresponds to $2 \mathrm{pA} / \sqrt{\mathrm{Hz}}$, i.e., one order of magnitude larger than the shot noise limit expected from values of $I_{\mathrm{dc}}$ of about $100 \mathrm{nA}$, typical of our experimental conditions. The additional noise appears to be due to instabilities in the $\mathrm{x}$-ray beam intensity. The signal amplitude was found to increase linearly with applied microwave power $\left(\propto B_{1}^{2}\right)$, as expected for $B_{1} \ll \Delta B_{0}$. Augmenting $\mathbf{B}_{1}$ represents the most straightforward way to improve the signal-to-noise ratio. Moreover, we found that $I_{\mathrm{dc}}$ and $I_{\mathrm{ac}}$ increase linearly with the beam intensity whereas the noise level is proportional to $\sqrt{I_{\mathrm{dc}}}$. Therefore, the signalto-noise ratio can be ameliorated also by maximizing the beam intensity and the solid angle covered by the photodiode.

To completely rule out the presence of spurious signals, such as inductive coupling between the photodiode and the resonator or field-modulated noise from stray photoelectrons emitted from the sample, we have performed a series of control experiments as reported in Fig. 4. We observe that: (1) When the x-ray beam is shut off and (2) when the photon energy is chosen in a spectral region where the dichroism is weak, no signal is detected above the noise level; (3) the XFMR signal disappears if $\mathbf{B}_{1}$ is turned off; and (4) the XFMR spectra are similar, but not identical to the FMR spectra simultaneously detected by measuring the microwave power reflected from the resonator by conventional methods. The latter effect is attributed to the difference between surface- and bulk-sensitive measurements due to the $\mathrm{x}$-ray absorption length in this energy range $(\sim 20 \mathrm{~nm}) .^{22}$

Given the XAS surface sensitivity, XFMR can be applied to samples with thickness down to a few tens of nm without considerable loss of signal. This makes it straightforward to extend XFMR to metallic samples where the skineffect limits the penetration of microwave radiation. Future experiments will test the possibility to perform XFMR using the photoelectron yield rather than fluorescence yield detection to further augment the surface sensitivity of the technique. X-ray detection of electron paramagnetic resonance, e.g., on metallorganic compounds, constitutes a possible extension of this technique.

The authors wish to thank G. Retout and N. B. Brookes for their help in preparing the experimental apparatus, $\mathrm{M}$. Fazan for cutting the YIG samples, ATC Europe (Stockholm, Sweden) for the supply of microwave capacitors, and Pacific Ceramics (Sunnyvale, CA) for providing the YIG specimens.

${ }^{1}$ B. Heinrich and J. Cochran, Adv. Phys. 42, 523 (1993).

${ }^{2}$ M. Farle, Rep. Prog. Phys. 61, 755 (1998).

${ }^{3}$ Z. Zhang, P. Hammel, and P. Wigen, Appl. Phys. Lett. 68, 2005 (1996).

${ }^{4}$ K. Wago, D. Botkin, C. Yannoni, and D. Rugar, Appl. Phys. Lett. 72, 2757 (1998)

${ }^{5}$ S. Tamaru, J. Bain, R. van de Veerdonk, T. Crawford, and M. Kryder, J. Appl. Phys. 91, 8034 (2002).

${ }^{6}$ R. Meckenstock, M. Möller, and D. Spoddig, Appl. Surf. Sci. 86, 112506 (2005).

${ }^{7}$ B. Hillebrands and K. Ounadjela eds., Spin Dynamics in Confined Magnetic Nanostructures I and II, Springer Topics in Applied Physics Vols. 83 and 87 (Springer, Berlin, 2002).

${ }^{8}$ C. Kittel, Phys. Rev. 115, 1587 (1959).

${ }^{9}$ S. Reidy, L. Cheng, and W. Bailey, Appl. Phys. Lett. 82, 1254 (2003).

${ }^{10}$ J. Pelzl, R. Meckenstock, D. Spoddig, F. Schreiber, J. Pflaum, and Z. Frait, J. Phys.: Condens. Matter 15, S451 (2003).

${ }^{11}$ J. Lindner and K. Baberschke, J. Phys.: Condens. Matter 15, S465 (2003).

${ }^{12}$ B. Heinrich, Y. Tserkovnyak, G. Woltersdorf, A. Brataas, R. Urban, and G. Bauer, Phys. Rev. Lett. 90, 187601 (2003).

${ }^{13}$ S. Kiselev, J. Sankey, I. Krivorotov, N. Emley, R. Schoelkopf, R. Buhrman, and D. Ralph, Nature (London) 425, 380 (2003).

${ }^{14}$ W. Rippard, M. Pufall, S. Kaka, S. Russek, and T. Silva, Phys. Rev. Lett. 92, 027201 (2004).

${ }^{15}$ M. Bonfim, G. Ghiringhelli, F. Montaigne, S. Pizzini, N. Brookes, F. Petroff, J. Vogel, J. Camarero, and A. Fontaine, Phys. Rev. Lett. 86, 3646 (2001).

${ }^{16}$ W. Bailey, L. Cheng, D. Keavney, C. Kao, E. Vescovo, and D. Arena, Phys. Rev. B 70, 172403 (2004).

${ }^{17}$ J. Vogel, W. Kuch, J. Camarero, K. Fukumoto, Y. Pennec, S. Pizzini, M. Bonfim, F. Petroff, A. Fontaine, and J. Kirschner, Phys. Rev. B 71, 060404(R) (2005).

${ }^{18}$ J. Stöhr, J. Magn. Magn. Mater. 200, 470 (1999).

${ }^{19}$ R. LeCraw, E. Spencer, and C. Porter, Phys. Rev. 110, 1311 (1958).

${ }^{20}$ P. David and M. Heath, J. Phys. C 3, 1356 (1970).

${ }^{21}$ G. van der Laan and B. Thole, Phys. Rev. B 42, 6670 (1990).

${ }^{22}$ S. Gota, M. Gautier-Soyer, and M. Sacchi, Phys. Rev. B 62, 4187 (2000). 